\title{
Four Disruptive Strategies for Removing Drug Discovery Bottlenecks
}

Sean Ekins ${ }^{1}{ }^{*}$, Chris L. Waller ${ }^{2,}{ }^{3}$, Mary P. Bradley ${ }^{4}$, Alex M. Clark ${ }^{5}$ and Antony J. Williams 6

${ }^{1}$ Collaborations in Chemistry, 5616 Hilltop Needmore Road, Fuquay-Varina, NC 27526, U.S.A.

${ }^{2}$ Pfizer Inc., Eastern Point Road, Groton, CT 06340.

${ }^{3}$ School of Pharmacy, University of North Carolina, Chapel Hill, NC 27514

${ }^{4}$ CollaborationFinder, Harvard Square, One Mifflin Place, Suite 400, Cambridge, MA 02138

${ }^{5}$ Molecular Materials Informatics, 1900 St. Jacques \#302, Montreal, Quebec, Canada H3J $2 \mathrm{~S} 1$.

${ }^{6}$ Royal Society of Chemistry, 904 Tamaras Circle, Wake Forest, NC 27587.

* To whom correspondence should be addressed.

Sean Ekins, E-mail address: ekinssean@ yahoo.com, Phone: +1 2156871320 


\begin{abstract}
Drug discovery is shifting focus from industry to outside partners and, in the process, creating new bottlenecks. Technologies like high throughput screening (HTS) have moved to a larger number of academic and institutional laboratories in the USA, with little coordination or consideration of the outputs and creating a translational gap. Although there have been collaborative public-private partnerships in Europe to share pharmaceutical data, the USA has lagged behind. Sharing precompetitive computational models may be the next frontier to provide confidence in the quality of leads produced and attract investment. We now suggest four disruptive strategies for removing drug discovery bottlenecks and discuss some novel ideas for the future to rectify the current unsustainable situation.
\end{abstract}




\section{HTS, Bottlenecks and Databases}

Bottleneck - any point at which movement or progress is slowed up because much must be funneled through it (Webster's New World College Dictionary).

Currently large pharmaceutical companies are undergoing (what appears to be) selective disintegration, while contract research organizations (CROs) and academia are growing in influence, publicly-funded drug development programs are expanding, precompetitive efforts are increasing and there is a re-emergence of venture-backed biotechnology firms [1]. These developments have created a dynamic ecosystem, with pharma as smaller nodes in a complex network in which collaborations have become an important business model. However, we are seeing a shift in focus away from early stage drug discovery, counter to what some have suggested is necessary for the industry to survive post disintegration [2]. This move away from early drug discovery is exemplified by the shift of high throughput screening (HTS) for drug discovery from a small number of major pharmaceutical companies to a larger number of academic and institutional laboratories in the USA. A pattern that is also repeating in Europe with closer academiaindustry ties [3]. To us, it seems this shift is counter intuitive and unknowingly creating a bottleneck by pushing a problem elsewhere. Some drugs, and a large percentage of leads, are discovered by companies using HTS [4], yet there are also examples in which HTS fails, in particular target-based antibacterial research and other areas [5,6]. Learning from the accumulated experience of pharmaceutical companies with HTS is instructive and may have been overlooked based on what we are seeing in the academic screening centers. A recent study surveyed 78 academic screening centers in the USA focused on 
high risk drug targets [7]. Results identified major gaps in efficacy testing, drug metabolism, PK studies and the challenge of translation to the clinic, commonly termed the "valley of death". These gaps, incidentally, are all skills that pharma has been rapidly removing and outsourcing. This leaves only CROs and clinically affiliated institutes able to overcome this bottleneck. Another issue identified by researchers from Bayer (which is receiving considerable attention) is that literature data on potential drug targets is often not reproducible [8]. Translating more compounds to the clinic from HTS screening centers without controlling for bias in pre-clinical proof-of-concept and target-based studies may also cause additional failures [9]. Taking HTS out of pharmaceutical companies has not so far achieved innovative breakthroughs. However, the government of the USA, through different agencies, is also heavily invested in the large HTS paradigm (paralleling that which was in industry) via such initiatives as ToxCast [10,11], Tox21[12], the Molecular Libraries Probe Production Centers Network (MLPCN) [13], the National Center for Translational Therapeutics (NCATS) [14], and the LINCS project [15] alongside the institutional screening centers, with little apparent coordination or consideration of the outputs. We also have concerns regarding simply using the HTS assays (and data) that were optimized to minimize "false negatives" for risk assessment purposes too, because these require minimizing false positives.

A crowdsourcing evaluation of MLPCN probes suggested to us that academic screening may result in a large number of dubious leads [13] and thereby creates another potential bottleneck. All of the screening efforts are generating very large quantities of data, and there is an expectation that it should be freely accessible. However, this would require databases that can handle structures and multiple bioactivity endpoints. Recent 
efforts funded by the US National Institutes of Health (NIH) with the NCATS Pharmaceutical Collection [16] (formerly the NCGC Pharmaceutical Collection (NPC) NPC browser) [17] suggest this is not straightforward [18,19], and poor data quality will severely impact the (cost effective but increasingly informatics-dependent) tools being used for drug repurposing efforts [20]. In our opinion, there needs to be independent assessment and curation of the HTS data produced across the board before embarking on more investments [19]. Efforts to carefully prepare deposition files and house high quality molecule and related data may look to ChemSpider [21,22] and ChEMBL[23], respectively as examples of how to do it. Without good databases for the HTS data yet another bottleneck is created.

It is also unclear how such HTS data is policed to make sure it goes out in a timely manner, and of the highest quality, and with maximal exposure. Although there is a requirement of all NIH-funded initiatives to outline a data sharing plan, we are not aware of any funding agency mandating data to be published along with 'quality guidelines', although we have suggested previously that granting bodies should have minimal quality standards for published data [18]. An extension to this would be that all data generated from publicly funded research should be openly available, within a year of generation, in high quality internet databases. To the best of our knowledge this is not something that is covered by current data sharing plans by most major funding agencies. Quality and accessibility of data will become increasingly important as there is more emphasis on academia for early drug discovery. The use of good bioassay ontologies could help in the standardization of this data. Recent efforts such as BioAssay Ontology 
[24] have shown the benefits for annotating, querying and analyzing such data, but these ontologies are yet to be widely applied.

We think part of the current trend in terms of proliferation of HTS screening initiatives is due to lack of coordination of government agencies, creating duplication and overlap, as exemplified by numerous chemical databases in North America containing approved drugs (Table 1). The government agencies would argue that redundancy in funding mitigates risk; however, if there is no sharing of data or experience ex post facto, then the risk of duplicative failure and unproductive expenditure increases. From what we see, there is too little collaboration around databases, curation, data quality [18] or even openness of the data across our domain. It is however acknowledged that data licensing is a complex issue and suggested approaches to Open Data licensing are under discussion [25].

There has been considerable discussion in the context of NCATS, about the urgent need to revamp how drugs are developed and brought to market faster and what incentives can be provided to generate treatments for neglected and rare diseases [14]. We question however whether any government or academic institute, as they currently stand, can adequately pursue such goals when an entire industry is struggling with the same challenges and has not come up with a solution. Many of the techniques proposed by NCATS [14], just like HTS, will not dramatically impact the process alone because this has not occurred in pharma. For example, elegant high-throughput screens at NCATS using the Pharmaceutical Collection of nearly 3000 drugs, identified 3 molecules as potential modulators of peripheral myelin protein 22 [26], yet it is unclear how these 
molecules (which all upregulated c-jun and therefore had potential for adverse central nervous system effects) would be used as potential treatments.

The misuse or non-use of HTS data (whether from pharma or academic centers) has been a problem since the inception of the technology. Simply because data exist somewhere, in a repository that is open, does not imply that the data are readily available or in a format that is fit for any given purpose (one could also say the same of the massive microarray databases). Achieving a positive return on any investment in HTS, or for that matter any potential drug discovery technology relies on producing available, usable, and complete data.

This leads us to propose what is needed for Disruptive Strategy \#1: NIH and other international scientific funding bodies should mandate minimum data quality standards, strict timelines for data submission, and open accessibility for all data generated by publicly funded research. We would also add that manuscripts resulting from NIH funded research (intra- and extramural) and public funded research in general, should also be immediately freely accessible if we are to prevent yet another bottleneck in transfer of knowledge. If enacted this will ensure that at the very least NIH (and other organizations) funded and published data, is of a high standard and that it would be readily accessible to a large audience. If the large population of NIH funded researchers can lead the way it is likely that other scientists will follow (e.g. consider how the deposition of protein structures in the Protein Databank is a requirement for most journals). There should be as few barriers to knowledge transfer as possible and information funded by the taxpayer in any country should be readily accessible as soon as possible. 
Collaboration and Public Private Partnerships (PPPs) The demand for companies to create value, combined with the increasing complexity of research, makes collaboration a reality that can no longer be denied. This begs the question of how we can remove the bottlenecks impeding progress in collaboration now. Academic groups could avoid the "valley of death" by working more closely with CROs and virtual pharma companies to do more preclinical and development studies, who in turn will work with pharma companies to purchase the most promising compounds. To do this there needs to be an awareness of what research is going on in the screening centers, and they in turn should be aware of groups that can take their screening hits and pursue them.

There is general agreement that the key to breakthrough success is collaboration [27]. There is also consensus that social networking can provide an effective platform for increasing collaboration in biomedical research [28]. However, to date, this has failed to take hold. The reason is fundamental: monetization of intellectual property (IP). There is no incentive for research organizations to disclose their current research in an open social networking forum where competitors have equal access. This is even true in academic research where investigators compete for funding. The key to success of this model of collaboration is the security of IP and the ability to selectively disclose IP to a valid potential partner in a secure way that results in a mutually equitable outcome for all parties [29]. Research collaborations are currently most advanced in the areas of neglected diseases, where funding comes primarily from public sources, data is more open, and potential profits are low or nil. The same situation is true for rare diseases $[30,31]$ and one would expect the creation of networks and ways to do more with less funding using collaborative software [27,32] will be essential. In both neglected and rare 
diseases, the partners are more likely to share IP because the monetary value of the IP ceases to be a barrier. Already there are several software products and initiatives which can be used to enable collaboration while retaining IP, these include CDD [33] and HEOS [32] which are used in major collaborative projects for tuberculosis and malaria research, respectively. But this begs the question why do we need to bother about IP at such early stages of drug discovery because it also creates a bottleneck.

There is potentially an alternative approach that ignores the IP associated with early research in an effort to make drug discovery more open, analogous to open source software [34]. Alongside the increasing mobility of computers the shift to mobile applications (apps) presents an opportunity to impact drug discovery [35] and specifically create the Open Drug Discovery Teams (ODDT) project. The project includes an app that has been created for the iOS to take advantage of the pharmaceutical data appearing in social media such as Twitter and Google Alerts which includes experimental data, molecule structures, images and other information that could be used for drug discovery collaborations. This app can be used by scientists as well as the public to follow a research topic by its hashtag or search terms, potentially publish data and share their ideas in the open. Already this app has been used by the rare disease community to share information.

Given that research organizations appear to be more receptive to embracing a new paradigm of collaboration, how is one scientist to know what other work is currently ongoing in a specific therapeutic or disease area when this is private? The key areas for success in biomedical research collaborations are for organizations to be able to "identify best-in-class capability, evaluate opportunities presented by programs and understand the 
associated risks" [36]. Currently, there is a lack of support mechanisms to identify and foster collaborations, resulting in a time consuming hit-or-miss process that relies on networking, internet searching, and attending scientific conferences. New services that provide a low cost, efficient means of finding targeted scientific connections for research and funding, while protecting IP will be key to connect everyone with a role in drug discovery and development. Because academic drug discovery centers and virtual companies will have nowhere near the resources or experience of a big pharma, much more work will need to be performed in silico [20] as well as in a collaborative manner [27] to ensure likely success. Another way to look at this is that a new virtual team paradigm has the potential to innovate through disruption.

There have been several collaborative PPPs in Europe to share drug safety data [37], ontologies and models [38] and knowledge management of pharmacological data $[39,40]$, all of which foster collaboration, as well as data sharing from industry and academia. In comparison, the USA has few comparable efforts currently ongoing in its research portfolio (e.g. Sage BioNetworks Arch2POCM to look at therapeutic targets [41]) and has barely embraced PPPs to the same extent as Europe. The reader is referred to a more extensive reviews of PPPs elsewhere [42,43]. However, such shared knowledge could help virtual pharmas, academics and institutes alongside pre-competitive initiatives [43,44] like those in informatics e.g. Pistoia Alliance [45], BioIT Alliance[46], PRISME [47] and W3C [48], to focus on the best ideas. The key challenge here is to ensure the delivery of tools or services to solve common problems for all parties involved and that there is coordination, progress and no overlap with the PPP initiatives described above. All of these efforts work by lowering the cost of research and remove duplication of 
efforts. A direct example is the structure representation standards documented for the FDA's substance registration system [49] whose recommendations have largely been adopted by ChEMBL [23] and will be implemented into ChemSpider [50] to support the OpenPHACTS project [51] for pharmaceutical companies that are participating in this initiative [19].

One additional way to lessen or remove existing barriers to collaboration might be to create a forma Japan [52]. For example, the major companies in a Japanese industry will come together with relevant government agencies and research organizations to develop plans, skills, technology, infrastructure, tooling, etc. that support the competence of that industry. It is only after this basic competence has been built that the companies resume competition with each other. The global automotive industry has undergone a similar transformation with increased pre-competitive collaboration [53] since 2003 (while retaining profitability) and today, automotive suppliers account for 50 percent of R\&D spending and 75 percent of the vehicle manufacturing. Similar pre-competitive collaborations occur in the semiconductor industry too [54]. Therefore, the relative resistance to drug discovery related research PPPs in the US is unlikely to be due to cultural differences because they occur in other industries.

This leads us to propose what is needed for Disruptive Strategy \#2: Reboot the pharmaceutical industry by extending the notion of "pre-competitive" collaboration to encompass later stages of research to allow PPPs to flourish. The role of large pharma is late stage development and branding, not early $R \& D$. If enacted this strategy will encourage more early stage collaboration between different stakeholders (multiple 
companies, academics and other parties), reduce duplication of efforts within companies, enhance the sharing of best-practices and resources around drug discovery and ensure that there is a focus on producing the best candidate for a disease or disease target.

\section{Translational Informatics}

As big pharma relies more on the CROs and academics, they will focus on translational informatics (integrated software solutions to manage the logistics, data integration and collaboration) and other efforts later in clinical development (such as Pfizer's ePlacebo) and post-marketing. This latter example uses placebo dosing data from previously executed clinical trials to augment or potentially supplant the need for placebo control groups in clinical trials. Expanding this to a larger scale, forming a cross-pharma data sharing consortium would dramatically impact the cost associated with clinical trial recruitment and execution of placebo dosing. However, this has not occurred yet. In an effort to stimulate data sharing of this type the FDA has announced an overhaul of its IT infrastructure [55]. A first step is the effort to make the historical clinical data in the FDA's vaults public. This is to be followed by a vast amount of de-identified post-market surveillance data. By doing this, the FDA hopes that the open data movement will stimulate the creation of PPPs aimed at sharing data relevant to other drug development stages. The FDA could go further and mandate all de-identified clinical data be made

public as part of the cost of doing business. Although some groups are pro [56] and others con [57], this approach could be universally useful for health research. It will also be important that we learn from clinical failures as well as successes [58]. We should be 
aware of potential barriers to data sharing and collaboration. Data and information silos exist at all levels of organizations. Allowing for data/information integration across silos is not a technological problem, regardless of issues of taxonomies and ontologies, but those will be much easier to surmount than the cultural, societal, and behavioral barriers to effective collaboration [27]. Such non-technical issues generally inhibit translational data analysis on a broad scale. With all the distributed research efforts we do not want to see creation of new data silos across the industry or in academia.

This leads us to propose what is needed for Disruptive Strategy \#3: FDA takes a proactive role in making available relevant clinical data that will help NCATS to bridge the valley of death and remove a bottleneck. If enacted this will ensure that companies and academia could also learn from the clinical data, build models and improve predictive tools (e.g. toxicity models like hERG [59], drug induced liver injury [60,61] etc).

\section{Mining by swarm and finding the best collaborators}

The FDA and the National Health Service (NHS) [62] have recently discussed the 'big data' or 'analytics' future involving analysis of patient data. This is only part of the picture in our opinion. There is an untapped source of data and that is the social media that is growing exponentially and has been used for disease surveillance $[63,64]$ and predicting health intervention [65] for example. The crowd is currently unwittingly providing us with valuable data (which we are not capturing and saving) that can be readily extracted from the web and social networks. We are therefore moving into the era 
of drug safety analysis, drug repurposing and marketing by sentiment analysis using social media stream mining tools such as Teranode [66], Ceiba [67], and Swarmology [68]. Swarm intelligence is a new subfield of bio-inspired artificial intelligence that offers solutions to complex problems such as pooled health-related data from different organizations as well as real-time data from social networks [69]. Emerging and likely disruptive technologies described above that listen to the crowd passively do not appear to be on the agenda at the FDA [55].

At the same time, the fringes of industry and academia may harbor the real innovators that should be funded to transform $R \& D$. Both governments and pharma companies could use software like Collaboration Finder [70] to find the best researchers to fund and collaborators to work with on strategic priorities (Figure 1). This would enable the NIH or other funding bodies to fund continuous innovation, rather than rebuilding academia in the shape of big pharma. Use of tools for collaboration, whether open like ODDT or those that maintain IP should also be applied. Disruption of the pharmaceutical industry may also begin with a fundamental rethinking of how to reward collaborative researchers in any organization. This may be facilitated by using collaborative informatics tools and considering how multi-player collaborative computer games reward their participants for contribution, something we are seeing in crowdsourcing efforts [71,72].

This leads us to propose what is needed for Disruptive Strategy \#4: Companies listen to the patients as they generate data on social media, mine it for indications and off target effects. Companies and funding bodies will find the innovators to exploit this information and use collaborative tools to share data. If enacted this will ensure that new treatments 
are perhaps identified by the patients themselves, the best researchers are connected to work on problems that are funded by companies, funding bodies, or disease organizations and ultimately the data created is in a format that can be readily shared upon publication and reused.

\section{Concluding remarks}

In summary, if we are to remove drug discovery bottlenecks (and we have described several types), there are many related steps we can take. For example, we need to provide more confidence that lead compounds will have efficacy in vivo and be safe. Some of these aspects could be addressed using predictive models already assembled and exclusive to the pharmaceutical companies. Sharing pre-competitive data and computational models for ADME/Tox [73-75], whether through a PPP or collaborations, could ultimately provide more confidence in the quality of the leads produced in academia, such that they will attract investment by pharmaceutical companies. The approaches we have seen taken so far by the pharma companies, NIH, FDA [76] and others to try to improve R\&D have tried to address individual parts of the process, when in fact there needs to be a complete overhaul and rethink akin to our workflow (which admittedly only covers the collaboration, data quality and openness aspects (Figure 1)). With new mobile computer hardware and software applications aspects of drug discovery will be done anywhere by potentially anyone [71] and this will be performed in a collaborative manner [77] so apps will need to be loosely 'connected' to create workflows [78]. If we are to facilitate this we need to ensure that data that is used that has 
been published by others is accessible (machine readable) and that it is of a high standard in the various databases.

We think it is time for disruption across the board and we suggest here that there are emerging technologies that could be used to reimagine drug discovery now (Figure 1).

\section{Acknowledgments:}

SE kindly acknowledges discussions with many collaborators including Mr. Bernard Munos, Dr. Joel Freundlich, Dr. Josef Scheiber and Ms. Julie Bryant.

\section{Conflicts of Interest:}

SE Consults for Collaborations in Chemistry, Collaborative Drug Discovery, Phoenix Nest, BioGan, Social Scientific Media and is on the board of directors for the Pistoia Alliance, AJW is employed by the Royal Society of Chemistry which owns ChemSpider and associated technologies and is involved with the Open PHACTS project. CW is an employee of Pfizer, MB is an employee of CollaborationFinder. AMC is the owner of Molecular Materials Informatics, Inc., and co-developed ODDT with SE. 


\section{References}

1 Robertson, G.M. and Mayr, L.M. (2011) Collaboration versus outsourcing: the need to think outside the box. Future Med Chem 3 (16), 1995-2020

2 Paul, S.M. et al. (2010) How to improve R\&D productivity: the pharmaceutical industry's grand challenge. Nat Rev Drug Discov 9 (3), 203-214

3 Academia-pharma collaboration attracts £14.4million funding in the UK to accelerate drug discovery. http://www.europeanpharmaceuticalreview.com/12777/news/industrynews/academia-pharma-collaboration-attracts-14-4million-funding-in-the-uk-toaccelerate-drug-discovery/

4 Macarron, R. et al. (2011) Impact of high-throughput screening in biomedical research. Nat Rev Drug Discov 10 (3), 188-195

5 Payne, D.A. et al. (2007) Drugs for bad bugs: confronting the challenges of antibacterial discovery. Nat Rev Drug Disc 6, 29-40

6 Rishton, G.M. (2005) Failure and success in modern drug discovery: guiding principles in the establishment of high probability of success drug discovery organizations. Med Chem 1 (5), 519-527

7 Frye, S. et al. (2011) US academic drug discovery. Nat Rev Drug Discov 10 (6), $409-410$

8 Prinz, F. et al. (2011) Believe it or not: how much can we rely on published data on potential drug targets? Nat Rev Drug Discov 10 (9), 712

9 Lindner, M.D. (2007) Clinical attrition due to biased preclinical assessments of potential efficacy. Pharmacol Ther 115 (1), 148-175 
10 Dix, D.J. et al. (2007) The ToxCast program for prioritizing toxicity testing of environmental chemicals. Toxicol Sci 95 (1), 5-12

11 Judson, R. et al. (2009) The toxicity data landscape for environmental chemicals. Environ Health Perspect 117 (5), 685-695

12 Tox21. http://www.rsc.org/chemistryworld/News/2011/December/chemicaltoxicity-human-health-testing.asp

13 Oprea, T.I. et al. (2009) A crowdsourcing evaluation of the NIH chemical probes. Nat Chem Biol 5 (7), 441-447

14 Collins, F.S. (2011) Reengineering translational science: the time is right. Sci Transl Med 3 (90), 90cm17

15 Lincsproject. http://www.lincsproject.org

16 NCATS Pharmaceutical Collection. http://www.ncats.nih.gov/research/tools/preclinical/npc/pharmaceutical$\underline{\text { collection.html }}$

17 Huang, R. et al. (2011) The NCGC Pharmaceutical Collection: A Comprehensive Resource of Clinically Approved Drugs Enabling Repurposing and Chemical Genomics. Sci Transl Med 3 (80), 80ps16

18 Williams, A.J. and Ekins, S. (2011) A quality alert and call for improved curation of public chemistry databases. Drug Disc Today $16,747-750$

19 Williams, A.J. et al. (2012) Towards a Gold Standard: Regarding Quality in Public Domain Chemistry Databases and Approaches to Improving the Situation. Drug Disc Today 17, 685-701 
20 Ekins, S. et al. (2011) In silico repositioning of approved drugs for rare and neglected diseases. Drug Disc Today 16, 298-310

21 ChemSpider. http:/www.chemspider.com

22 Pence, H.E. and Williams, A.J. (2010) ChemSpider: An Online Chemical Information Resource. J Chem Educ 87, 1123-1124

23 ChEMBL. http://www.ebi.ac.uk/chembldb/index.php

24 Visser, U. et al. (2011) BioAssay Ontology (BAO): a semantic description of bioassays and high-throughput screening results. BMC Bioinformatics 12, 257

25 Williams, A.J. et al. (2012) Why Open Drug Discovery Needs Four Simple Rules for Licensing Data and Models. PLoS Comput Biol In Press

26 Jang, S.W. et al. (2012) Identification of Drug Modulators Targeting GeneDosage Disease CMT1A. ACS Chem Biol

27 Ekins, S. et al. (2011) Collaborative computational technologies for biomedical research, Wiley

28 Gewin, V. (2010) Collaboration: Social networking seeks critical mass. Nature 468, 993-994

29 Bunin, B.A. and Ekins, S. (2011) Alternative business models for drug discovery. Drug Disc Today 16, 643-645

30 Griggs, R.C. et al. (2009) Clinical research for rare disease: opportunities, challenges, and solutions. Mol Genet Metab 96 (1), 20-26

31 Brewer, G.J. (2009) Drug development for orphan diseases in the context of personalized medicine. Transl Res 154 (6), 314-322 
32 Bost, F. et al. (2010) Informatics for neglected diseases collaborations. Curr Opin Drug Discov Devel 13 (3), 286-296

33 Hohman, M. et al. (2009) Novel web-based tools combining chemistry informatics, biology and social networks for drug discovery. Drug Disc Today 14, $261-270$

34 Williams, A.J. et al. (2011) Current and future challenges for the collaborative computational technologies for the life sciences. In Collaborative computational technologies for biomedical research (Ekins, S. et al., eds.), pp. 491-517, Wiley and Sons

35 Williams, A.J. et al. (2011) Mobile apps for chemistry in the world of drug discovery. Drug Disc Today 16, 928-939

36 Jones, A. and Clifford, L. (2005) Drug discovery alliances. Nat Rev Drug Discov $4(10), 807-808$

37 eTox. http://www.e-tox.net/index.html

38 Hardy, B. et al. (2010) Collaborative development of predictive toxicology applications. J Cheminform 2 (1), 7

39 Azzaoui, K. et al. (2012) Analysis of the scientific competency questions followed by the IMI OpenPHACTS consortium for the development of the semantic web-based molecular information system OPS. Drug Disc Today Submitted

40 Williams, A.J. et al. (2012) Open PHACTS: Semantic interoperability for drug discovery. Drug Disc Today In press 
41 Norman, T.C. et al. (2011) Leveraging crowdsourcing to facilitate the discovery of new medicines. Sci Transl Med 3 (88), $88 \mathrm{mr} 81$

42 Hunter, A.J. (2008) The Innovative Medicines Initiative: a pre-competitive initiative to enhance the biomedical science base of Europe to expedite the development of new medicines for patients. Drug Discov Today 13 (9-10), 371373

43 Hunter, J. (2011) Precompetitive collaboration in the pharmaceutical industry. In Collaborative computational technologies for biomedical research (Ekins, S. et al., eds.), pp. 55-84, Wiley and Sons

44 Norman, T. et al. (2011) The precompetitive space: time to move the yardsticks. Sci Transl Med 3 (76), 76cm10

45 Pistoia Alliance. http://pistoiaalliance.org/

46 BioIT Alliance. http://bioitalliance.org/

47 PRISME. http://prismeforum.org/

48 W3C. http://www.w3.org/

49 SRS.

http://www.fda.gov/ForIndustry/DataStandards/SubstanceRegistrationSystem$\underline{\text { UniqueIngredientIdentifierUNII/default.htm }}$

50 Williams, A.J. (2008) Internet-based tools for communication and collaboration in chemistry. Drug Discov Today 13 (11-12), 502-506

51 OpenPHACTS. http://www.openphacts.org/

52 Gibson, D.V. and Rogers, E.M. (1994) R\&D Collaboration on Trial, Harvard Business Review Press 
53 CLEPA, The European Association of Automotive Suppliers http://www.clepa.eu

54 Collaborative $\quad$ Alliance for $\quad$ Semiconductor $\quad$ Test http://www.semi.org/en/industrysegments/test/cast/ctr_029261

55 FDA. (2011) Advancing regulatory science at FDA U.S. Food and Drug Administration

56 Monash

research guidelines. http://www.researchdata.monash.edu/guidelines/deposit.html

57 Silversides, A. (2011) Withdrawal of clinical trials policy by Canadian research institute is a "lost opportunity for increased transparency". BMJ 342, d2570

58 Chiou, J.-Y. et al. (2012) The value of failures in pharmaceutical R\&D. IMT LUCCA EIC Working paper series \#01/2012 January, 1-26

59 Crumb Jr, W.J. et al. (2006) Effects of antipsychotic drugs on $\mathrm{I}_{\mathrm{to}}, \mathrm{I}_{\mathrm{Na}}, \mathrm{I}_{\mathrm{sus}}, \mathrm{I}_{\mathrm{K} 1}$, and hERG: QT prolongation, structure activity relationship, and network analysis. Pharm Res 23, 1133-1143

6 Liu, Z. et al. (2011) Translating clinical findings into knowledge in drug safety evaluation--drug induced liver injury prediction system (DILIps). PLoS Comput Biol 7 (12), e1002310

61 Ekins, S. et al. (2010) A Predictive Ligand-Based Bayesian Model for Human Drug Induced Liver Injury. Drug Metab Dispos 38, 2302-2308

62 NHS. http://www.rsc.org/chemistryworld/News/2011/December/08121101.asp

63 Chew, C. and Eysenbach, G. (2010) Pandemics in the age of Twitter: content analysis of Tweets during the 2009 H1N1 outbreak. PLoS One 5 (11), e14118 
64 Signorini, A. et al. (2011) The use of Twitter to track levels of disease activity and public concern in the U.S. during the influenza A H1N1 pandemic. PLoS One 6 (5), e19467

65 Corley, C.D. et al. (2011) Predicting individual affect of health interventions to reduce HPV prevalence. Adv Exp Med Biol 696, 181-190

66 Teranode. http://www.teranode.com

67 Ceiba. http://www.ceibasolutions.com/solutions/\#social

68 Swarmology. http://www.swarmology.com/),

69 Martens, D. et al. (2011) Editorial survey: swarm intelligence for data mining. Mach Learn 82, 1-42

70 CollaborationFinder. $\underline{\text { www.collaborationfinder.com }}$

71 Cooper, S. et al. (2010) Predicting protein structures with a multiplayer online game. Nature 466 (7307), 756-760

72 GalaxyZoo. http://www.galaxyzoo.org/story

73 Gupta, R.R. et al. (2010) Using open source computational tools for predicting human metabolic stability and additional ADME/TOX properties. Drug Metab Dispos 38, 2083-2090

74 Spjuth, O. et al. (2010) Towards interoperable and reproducible QSAR analyses: Exchange of datasets. J Cheminform 2 (1), 5

75 Ekins, S. and Williams, A.J. (2010) Precompetitive Preclinical ADME/Tox Data: Set It Free on The Web to Facilitate Computational Model Building to Assist Drug Development. Lab on a Chip 10, 13-22 
76 FDA. (2004) Innovation stagnation: challenge and opportunity on the critical path to new medicinal products.

77 Ekins, S. et al. (2012) Open Drug Discovery Teams: A Chemistry Mobile App for Collaboration. Molecular Informatics In Press

78 Clark, A.M. et al. (2012) Redefining cheminformatics with intuitive collaborative mobile apps. Molecular Informatics In Press 
Figure 1. A schematic of how several recent informatics technologies could disrupt pharmaceutical research. Technologies like CollaborationFinder can enable researchers to find potential collaborators and funding agencies can better understand who they should seek out and fund. Data generated, whether high or low throughput should be checked to ensure structure and data integrity using free databases and tools like ChemSpider. For private collaborations in which IP needs to be retained, Collaborative Drug Discovery (CDD) enables data sharing and mining. Open data published via social networks and open databases could be collated and shared with mobile applications like Open Drug Discovery Teams (ODDT). Potential for connectivity (dotted lines) between tools is shown to illustrate potential workflows. The solid line represents a current two way connection between ChemSpider and CDD. 
Table 1. North American small molecule databases containing FDA approved drugs

\begin{tabular}{|c|c|c|c|}
\hline $\begin{array}{l}\text { Database } \\
\text { name }\end{array}$ & Funding & Content and details & URL \\
\hline PubChem & $\mathrm{NIH}$ & $\begin{array}{l}>30 \mathrm{M} \text { molecules includes } \\
\text { FDA approved drugs }\end{array}$ & $\begin{array}{l}\text { http://pubchem.ncbi.nlm.nih.g } \\
\text { ov/ }\end{array}$ \\
\hline $\begin{array}{l}\text { NPC } \\
\text { Browser }\end{array}$ & $\mathrm{NIH}$ & $\begin{array}{lrr}\text { 10,000 } & \text { compounds } \\
\text { includes } & \text { FDA } & \text { approved } \\
\text { drugs } & & \end{array}$ & http://tripod.nih.gov/npc/ \\
\hline ToxCast & EPA & $\begin{array}{l}>1000 \text { compounds includes } \\
\text { some drugs and drug like } \\
\text { molecules }\end{array}$ & http://epa.gov/ncct/toxcast/ \\
\hline DailyMed & FDA & $\begin{array}{l}>31,942 \text { labels }- \text { many } \\
\text { labels for the same drug }\end{array}$ & $\begin{array}{l}\text { http://dailymed.nlm.nih.gov/da } \\
\text { ilymed/about.cfm }\end{array}$ \\
\hline ChemIDplus & $\mathrm{NIH}$ & $\begin{array}{l}>\quad 295,000 \quad \text { structures } \\
\text { including many FDA small } \\
\text { molecule approved drugs }\end{array}$ & $\begin{array}{l}\text { http://chem.sis.nlm.nih.gov/ch } \\
\text { emidplus/ }\end{array}$ \\
\hline DrugBank & Canadian & $\begin{array}{l}6707 \text { drug entries including } \\
1436 \text { FDA-approved small } \\
\text { molecule drugs (this may be } \\
\text { underestimated). }\end{array}$ & http://www.drugbank.ca/ \\
\hline
\end{tabular}

